Supporting Information

\title{
Lithium Ion Disorder and Conduction Mechanism in $\mathrm{LiCe}\left(\mathrm{BH}_{4}\right)_{3} \mathrm{Cl}$
}

Young-Su Lee, ${ }^{*}{ }^{\dagger}$ Morten B. Ley, ${ }^{\ddagger}{ }^{\ddagger}$ Torben R. Jensen, ${ }^{\star}$ and Young Whan Cho ${ }^{\dagger}$

${ }^{\dagger}$ High Temperature Energy Materials Research Center,

Korea Institute of Science and Technology, Seoul 02792, Republic of Korea

${ }^{+}$Center for Materials Crystallography (CMC), Interdisciplinary Nanoscience Center

(iNANO) and Department of Chemistry, University of Aarhus,

Langelandsgade 140, DK-8000 Århus C, Denmark

$\S$ Max-Planck-Institut für Kohlenforschung,

Kaiser-Wilhelm-Platz 1, 45470 Mülheim an der Ruhr, Germany

* Corresponding author.

E-mail: lee0su@kist.re.kr 


\section{Synthesis}

For the synthesis of $\mathrm{LiCe}\left(\mathrm{BH}_{4}\right)_{3} \mathrm{Cl}$ and $\mathrm{LiCe}\left({ }^{11} \mathrm{BD}_{4}\right)_{3} \mathrm{Cl}$, the samples $\mathrm{CeCl}_{3}-\mathrm{LiBH}_{4}$ 1:3 and $\mathrm{CeCl}_{3}-\mathrm{Li}^{11} \mathrm{BD}_{4}$ 1:3 were ball-milled (BM) in repeated sequences of 2 min milling and 2 min breaks for 60 repetitions using a Fritsch Pulverisette 4 planetary mill under inert conditions (argon atmosphere) in $80 \mathrm{~mL}$ of tungsten carbide steel containers with tungsten carbide balls (o.d. $10 \mathrm{~mm}$ ). The sample to balls mass ratio was approximately 1:35 and the speeds of the main disk and planetary disks were 200 and $560 \mathrm{rpm}$, respectively. The manipulation of the samples were performed in a glove box with a circulation purifier maintained under an argon atmosphere with $<1$ ppm of $\mathrm{O}_{2}$ and $\mathrm{H}_{2} \mathrm{O}$. The starting chemicals were lithium borohydride, $\mathrm{LiBH}_{4}$ (assay 95\%, Sigma-Aldrich), lithium borodeuteride, $\mathrm{Li}^{11} \mathrm{BD}_{4}$ (assay 95\%, KatChem), and anhydrous cerium chloride, $\mathrm{CeCl}_{3}$ (assay 99.9\%, Sigma-Aldrich). All chemicals were used as received.

For the $\mathrm{CeBr}_{3}-\mathrm{LiCl}$ sample, anhydrous $\mathrm{CeBr}_{3}$ (assay $99.99 \%$, Sigma-Aldrich) and $\mathrm{LiCl}$ (assay $>98 \%$, Fluka) were used as received. About $4.2 \mathrm{~g}$ of the 1:1 mixture (in molar ratio) was ball-milled in a $50 \mathrm{ml}$ hardened steel jar with three $12.7 \mathrm{~mm}$ and twelve $7.9 \mathrm{~mm}$ diameter Cr-steel balls. The ball-milling was conducted using a planetary mill (Retsch PM 200) at 600 rpm for $200 \mathrm{~min}$. All the sample handling except ball milling was carried out in an argon-filled glove box where both $\mathrm{O}_{2}$ and $\mathrm{H}_{2} \mathrm{O}$ levels were maintained below 0.1 ppm. After ball-milling, mostly $\mathrm{CeBr}_{3}$ was seen in the X-ray diffraction data. X-ray diffraction data were obtained using a Bruker D8 Advance diffractometer with $\mathrm{Cu} \mathrm{K \alpha}$ radiation, $\lambda=1.5418 \AA$. An elevated temperature might be required to stabilize the hypothetical $\mathrm{LiCeBr}_{3} \mathrm{Cl}$. Therefore, to locate thermal events, differential scanning calorimetry using a Netzsch DSC214 Polyma was performed. However, no thermal event which could be linked to the formation of $\mathrm{LiCeBr}_{3} \mathrm{Cl}$ was observed. A melting appears around $420{ }^{\circ} \mathrm{C}$ and we tested whether the hypothetical 
compound could be directly formed from the melt: the sample was heat-treated at $450{ }^{\circ} \mathrm{C}$, cooled to room temperature, and then analyzed again with X-ray diffraction. Some unidentified peaks together with $\mathrm{CeBr}_{3}$ was found but they did not match the simulated pattern of $\mathrm{LiCeBr}_{3} \mathrm{Cl}$. We therefore concluded that the formation of $\mathrm{LiCeBr}_{3} \mathrm{Cl}$ is thermodynamically unfavorable. 
Figure S1. Li ion distribution for the image number 0 (left) and 18 (right) of the nudged elastic band calculation in Figure 2. Only the Li ions are shown for simplicity.
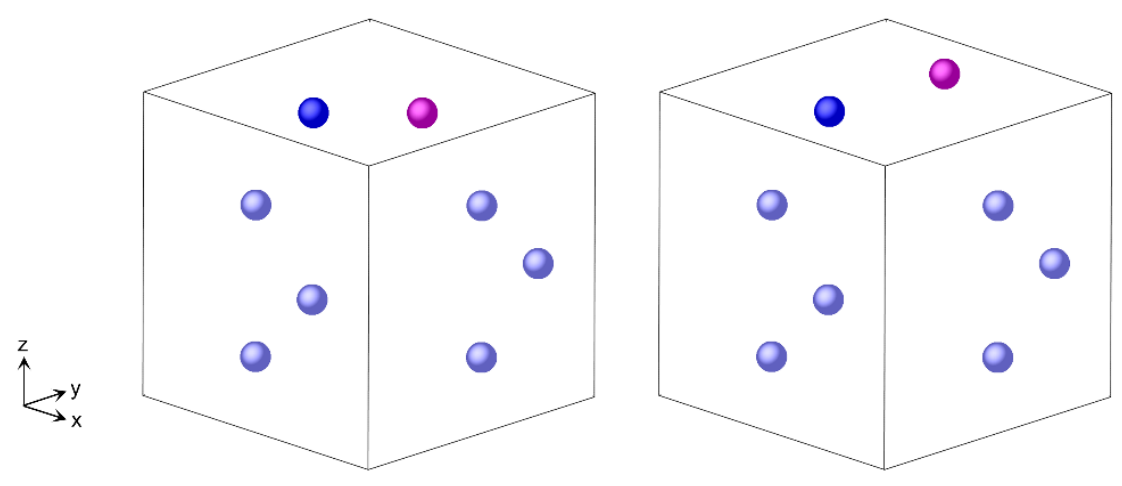
Figure S2. The polyhedral structure model of $\mathrm{LiCe}\left(\mathrm{BH}_{4}\right)_{3} \mathrm{Cl}$. Only a part of the sturcture is shown for a better visibility. Grey and green atoms are B and $\mathrm{Cl}$, respectively. The $\mathrm{T} 1$ and T2 tetrahderal sites and the octahedral site are displayed as blue, magenta, and green polyhedrons, respectively. The $\mathrm{T} 1$ tetrahedrons share a corner whereas the $\mathrm{T} 1$ and $\mathrm{T} 2$ tetrahedrons share an edge. The octahedral site shares its face with both the T1 and T2 sites.
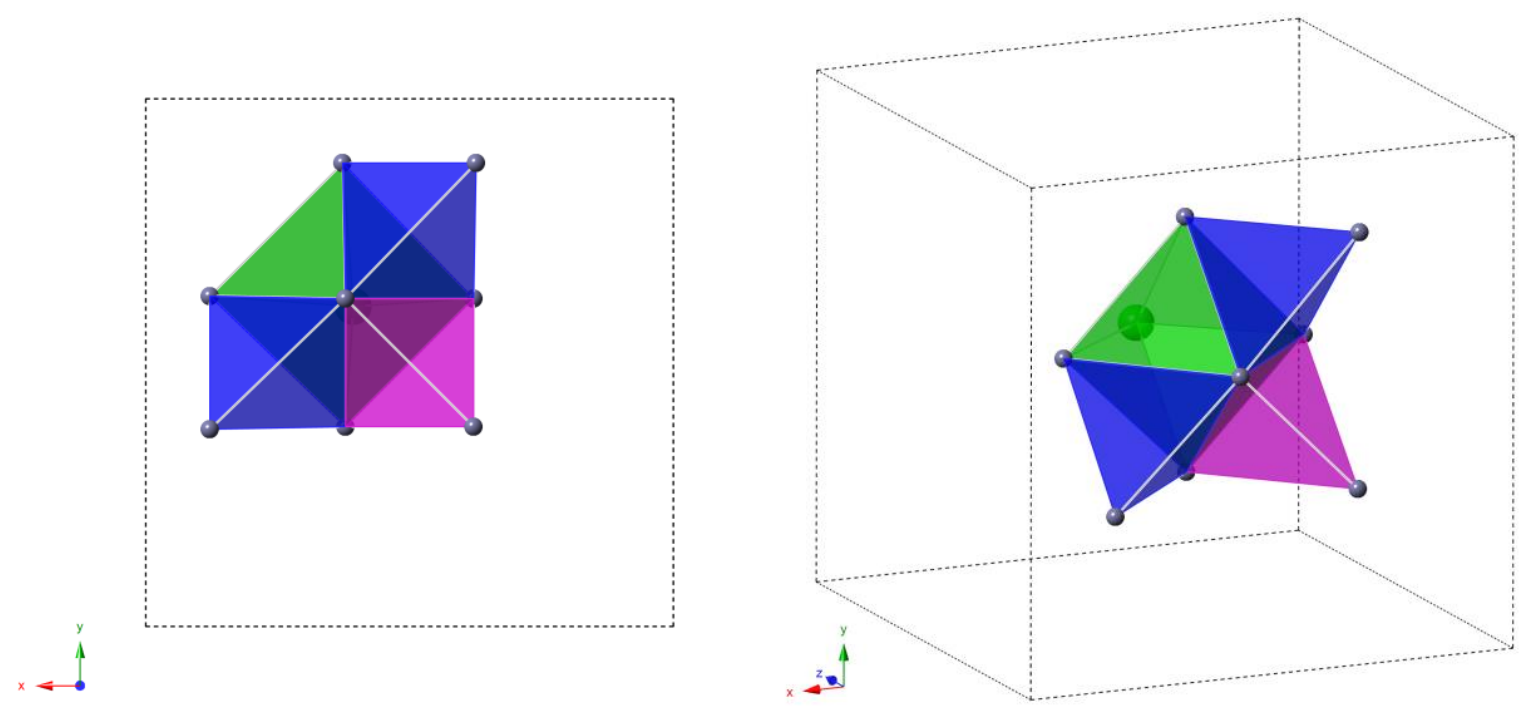
Figure S3. Li ion distribution for the image number 0 (left) and 18 (right) of the nudged elastic band calculation in Figure 4. Only the Li ions are shown for simplicity.
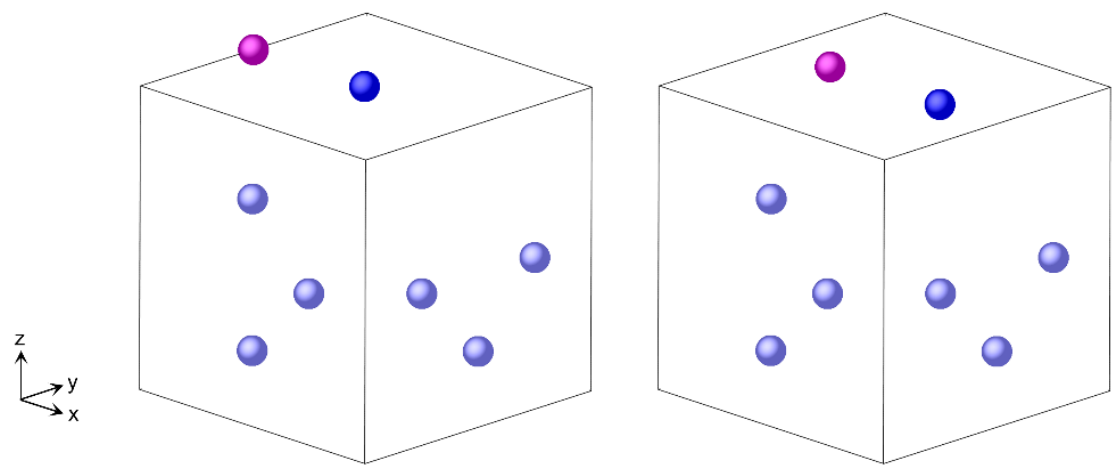
Figure S4. The charges for Li1 and Li2 along the trajectory of the Li1 jump (from image number 0 to 9 in Figure $4 \mathrm{a})$ in $\mathrm{LiCe}\left(\mathrm{BH}_{4}\right)_{3} \mathrm{Cl}$.

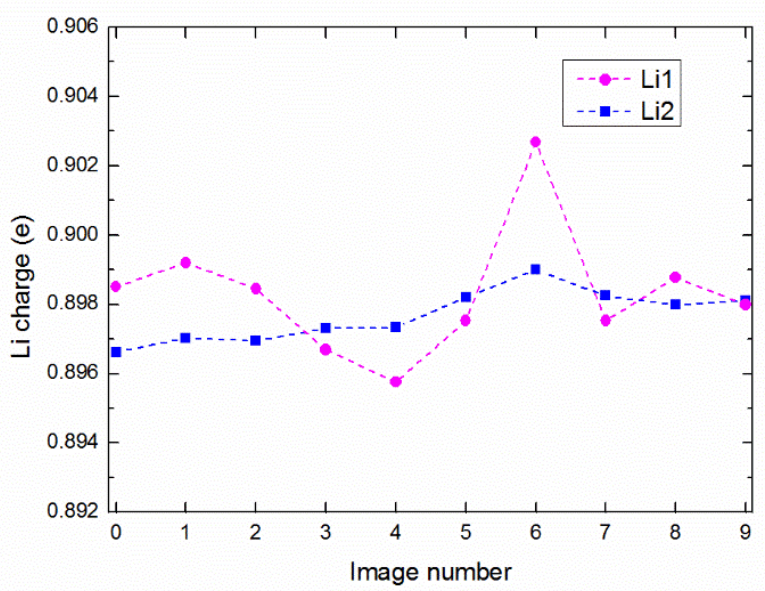


Figure S5. The network of combined $12 d$ (in blue) and $6 b$ (in magenta) Wyckoff sites in the $I-43 m$ space group. When the $24 g$ (in green) sites are also stable as in the hypothetical $\mathrm{LiCeBr}_{3} \mathrm{Cl}$, the jump distance for the $\mathrm{Li}$ ion diffusion will be further reduced by ca. $0.4 \AA$ with respect to the direct $12 d \leftrightarrow 6 b$ site jump.

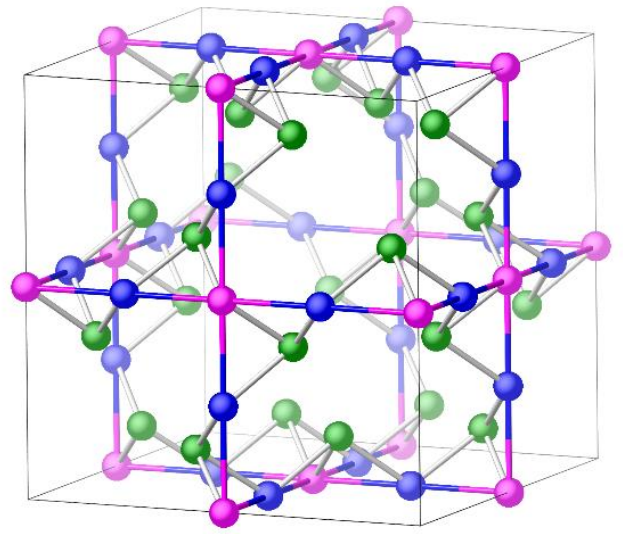


Figure S6. The mean square displacement (MSD) of the Li ions as a function of the MD simulation time. The MSD value is an average over the $32 \mathrm{Li}$ ions (four different starting configurations, $8 \mathrm{Li}$ ions per each configuration). The red dashed line is the linear interpolation of the data.

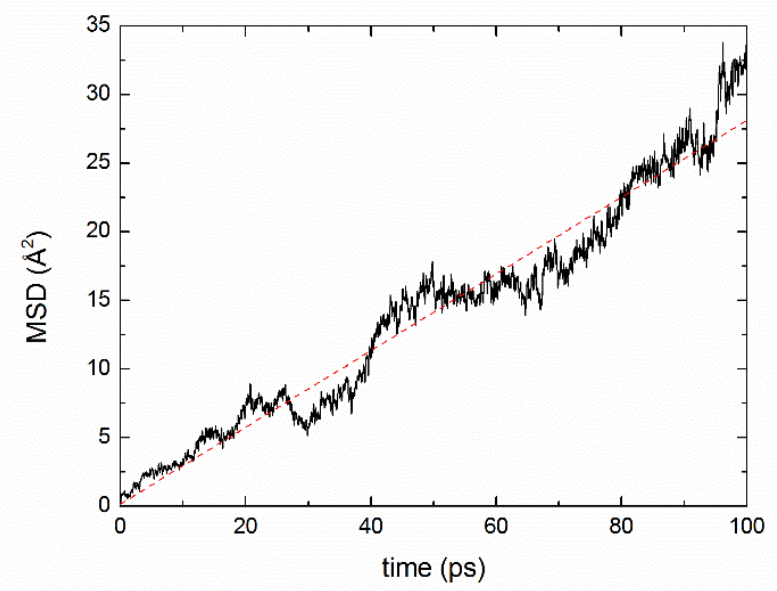


Figure S7. The evolution of the atomic configurations upon a $\mathrm{Li}$ ion jump. The seven configurations are snapshots at the time frames marked by arrows and coordination numbers in Figure 8a. The Li ion is colored in yellow and the indices for the $\left[\mathrm{BH}_{4}\right]^{-}$groups in the coordination shell are written in blue letters.
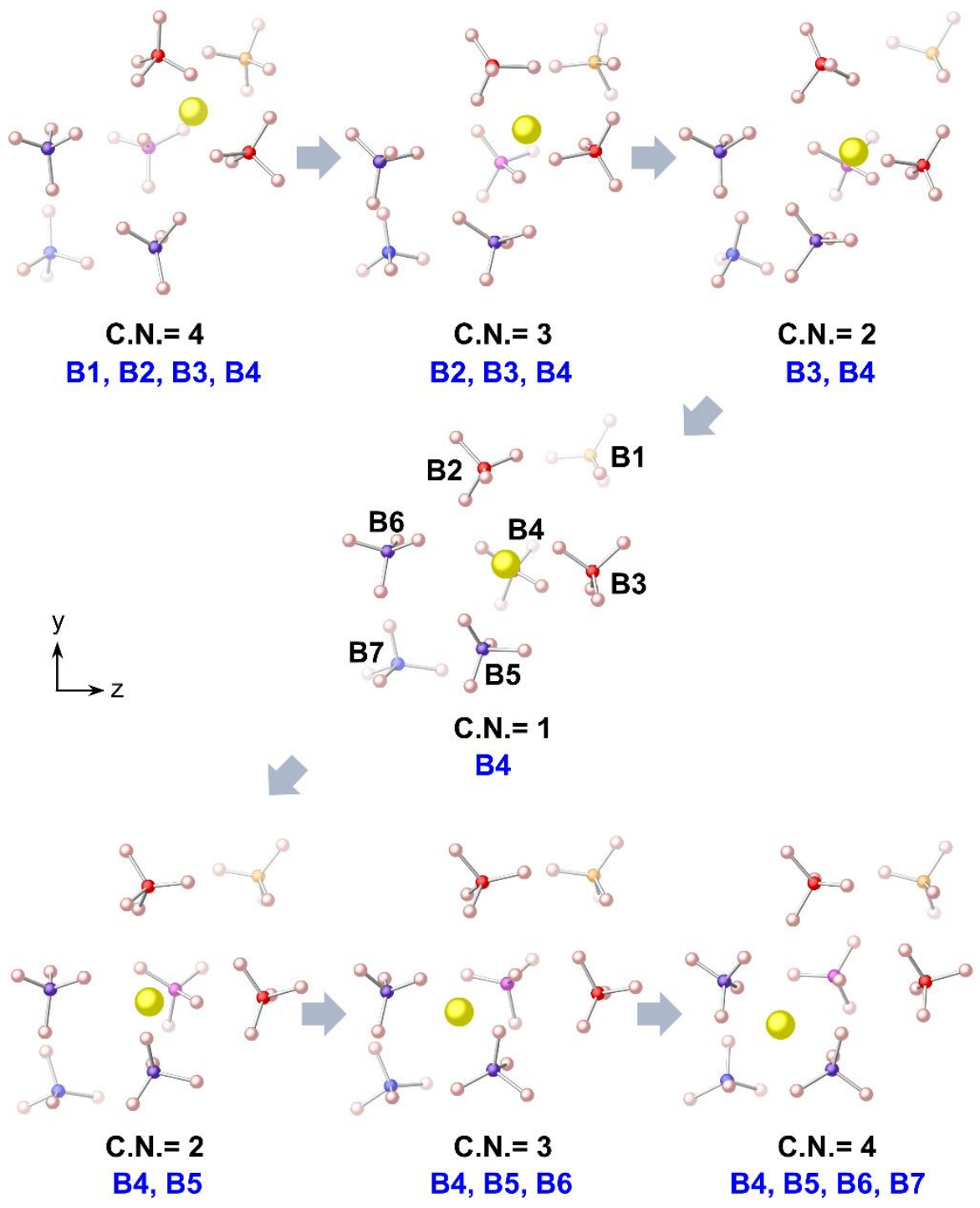